\title{
Pacific
}

Journal of

Mathematics

\section{BRAIDING KNOTS IN CONTACT 3-MANIFOLDS}

\section{Elena Pavelescu}




\title{
BRAIDING KNOTS IN CONTACT 3-MANIFOLDS
}

\author{
ELENA PAVELESCU
}

\begin{abstract}
We show that a transverse link in a contact structure supported by an open book decomposition can be transversely braided, and we generalize Markov's theorem on when the closures of two braids represent (transversely) isotopic links.
\end{abstract}

\section{Introduction}

Alexander [1923] proved that any knot in $\mathbb{R}^{3}$ can be braided about the $z$-axis. Bennequin, in a paper [1983] that marked the start of modern contact topology and led to the Bennequin inequality and eventually to Eliashberg's definition of tightness, proved the transverse case for $\left(\mathbb{R}^{3}, \xi_{\text {std }}\right)$. In this paper, we generalize Bennequin's result to any closed oriented 3-dimensional manifold $M$, by looking at an open book decomposition for $M$ together with its supported contact structure. After we completed the proof of this theorem, it was brought to our attention that the same result was independently obtained through different means by Mitsumatsu and Mori in [Mitsumatsu 2006].

Bennequin used braid theory in $\mathbb{R}^{3}$ to show the standard contact structure was tight. Since then, powerful analytic machinery such as holomorphic curves and Seiberg-Witten theory has been used to prove tightness. By studying braids in general open books, it is hoped that tight contact structures can be better understood from a purely topological/combinatorial perspective. The results presented in this paper constitute a first step in this program. While these results are purely geometric, it would be nice to have an algebraic description of a generalized "braid group". In particular, one needs to define the stabilization moves with respect to different binding components and account for the fundamental group of the page. We will explore this algebraic perspective in a subsequent paper.

Markov [1936] gave an equivalent condition for the closures of two braids in $\mathbb{R}^{3}$ to be isotopic as links, if and only if the two braids differ by conjugations in the braid group and positive and negative Markov moves. Orevkov and Shevchishin [2003] proved the transversal case for $\left(\mathbb{R}^{3}, \xi_{\text {std }}\right)$. Wrinkle [2002] independently obtained a different proof. We generalize Markov's theorem to any closed oriented

MSC2010: 57M50.

Keywords: braid, transverse link, open book decomposition. 
3-dimensional manifold. We prove the transverse case and recover the topological case proved in [Skora 1992] and [Sundheim 1993].

In [Kawamuro and Pavelescu 2011] the braiding results presented in this paper are used to find combinatorial self-linking formulae for null-homologous braids in annulus open book decompositions and pants open book decompositions, extending Bennequin's self-linking formula for a braid in the standard contact 3-sphere.

\section{Contact structures and open book decompositions}

Let $M$ be a compact, oriented 3-manifold, and $\xi$ an oriented 2-plane field on $M$. We say $\xi$ is a contact structure on $M$ if $\xi=\operatorname{ker} \alpha$ for some global 1-form $\alpha$ satisfying $\alpha \wedge d \alpha \neq 0$. In this paper we assume that the manifold $M$ is oriented and that the contact structure $\xi$ is oriented and positive (that is, $\alpha \wedge d \alpha>0$ ).

A contactomorphism between two contact manifolds $\left(M_{1}, \xi_{1}\right)$ and $\left(M_{2}, \xi_{2}\right)$ is a diffeomorphism $\phi: M_{1} \rightarrow M_{2}$ such that $\phi_{*} \xi_{1}=\xi_{2}$.

On $\mathbb{R}^{3}$, consider the two contact structures $\xi_{1}$ and $\xi_{2}$ given by the 1 -forms $\alpha_{1}=$ $d z-y d x$ and $\alpha_{2}=d z+r^{2} d \theta$ (given in cylindrical coordinates). Then $\left(\mathbb{R}^{3}, \xi_{1}\right)$ and $\left(\mathbb{R}^{3}, \xi_{2}\right)$ are contactomorphic and we refer to both $\xi_{1}$ and $\xi_{2}$ as the standard contact structure on $\mathbb{R}^{3}, \xi_{\text {std }}$.

Theorem 2.1 (Gray's theorem). Let $\left\{\xi_{t}\right\}_{t \in[0,1]}$ be a family of contact structures on a manifold $M$ that differ on a compact set $C \subset \operatorname{int}(M)$. Then there exists an isotopy $\psi_{t}: M \rightarrow M$ such that

(i) $\left(\psi_{t}\right)_{*} \xi_{1}=\xi_{t}$;

(ii) $\psi_{t}$ is the identity outside of an open neighborhood of $C$.

While the proof of this result is well-known, we sketch it here as we will need elements of it in later arguments. For more details see [Geiges 2008, p. 61].

Proof. We look for $\psi_{t}$ as the flow of a vector field $X_{t}$. If $\xi_{t}=\operatorname{ker} \alpha_{t}$, then $\psi_{t}$ has to satisfy

$$
\psi_{t}^{*} \alpha_{t}=\lambda_{t} \alpha_{0}
$$

for some nonvanishing function $\lambda_{t}: M \rightarrow \mathbb{R}$. By taking the derivative with respect to $t$ on both sides and rearranging the terms, we get

$$
\psi_{t}^{*}\left(\frac{d \alpha_{t}}{d t}+\mathscr{L}_{X_{t}} \alpha_{t}\right)=\frac{d \lambda_{t}}{d t} \alpha_{0}=\frac{d \lambda_{t}}{d t} \frac{1}{\lambda_{t}} \psi^{*} \alpha_{t}
$$

This is equivalent to

$$
\psi_{t}^{*}\left(\frac{d \alpha_{t}}{d t}+d\left(\iota_{X_{t}} \alpha_{t}\right)+\iota_{X_{t}} d \alpha_{t}\right)=\psi_{t}^{*}\left(h_{t} \alpha_{t}\right)
$$


for

$$
h_{t}=\frac{d}{d t}\left(\log \lambda_{t}\right) \circ \psi_{t}^{-1} .
$$

If $X_{t}$ is chosen in $\xi_{t}$, then $\iota_{X_{t}} \alpha_{t}=0$, and (2-3) becomes

$$
\frac{d \alpha_{t}}{d t}+\iota_{X_{t}} d \alpha_{t}=h_{t} \alpha_{t}
$$

Applying (2-4) to the Reeb vector field of $\alpha_{t}, v_{\alpha_{t}}$ (that is, the unique vector field $v_{t}$ such that $\alpha_{t}\left(v_{t}\right)=1$ and $\left.d \alpha_{t}\left(v_{t}, \cdot\right)=0\right)$, we find $h_{t}=\left(d \alpha_{t} / d t\right)\left(v_{\alpha_{t}}\right)$ and $X_{t}$ given by

$$
\iota_{X_{t}} d \alpha_{t}=h_{t} \alpha_{t}-\frac{d \alpha_{t}}{d t} .
$$

The form $d \alpha_{t}$ gives an isomorphism

$$
\Gamma\left(\xi_{t}\right) \rightarrow \Omega_{\alpha_{t}}^{1}, \quad v \mapsto \iota_{v} d \alpha_{t},
$$

where $\Gamma\left(\xi_{t}\right)=\left\{v \mid v \in \xi_{t}\right\}$ and $\Omega_{\alpha_{t}}^{1}=\left\{1\right.$-forms $\left.\beta \mid \beta\left(v_{t}\right)=0\right\}$, and thus $X_{t}$ is uniquely determined by (2-4). By construction, the flow of $X_{t}$ is the desired $\psi_{t}$. For the subset of $M$ where the $\xi_{t}$ agree we choose the $\alpha_{t}$ to agree. This implies that $d \alpha_{t} / d t=0$, $h_{t}=0$ and $X_{t}=0$, and all equalities hold.

In a contact manifold $(M, \xi)$, an oriented arc $\gamma \subset M$ is called transverse if for all $p \in \gamma$ and $\xi_{p}$ the contact plane at $p, T_{p} \gamma \pitchfork \xi_{p}$ and $T_{p} \gamma$ intersects $\xi_{p}$ positively. Also, if $\gamma$ is a closed curve then it is called a transverse knot.

An open book decomposition of $M$ is a pair $(\mathrm{L}, \pi)$ where

- $L$ is an oriented link in $M$ called the binding of the open book, and

- $\pi: M \backslash L \rightarrow S^{1}$ is a fibration whose fiber, $\pi^{-1}(\theta)$, is the interior of a compact surface $\Sigma \subset M$ such that $\partial \Sigma=L$, for all $\theta \in S^{1}$. The surface $\Sigma$ is called the page of the open book.

Alternatively, an open book decomposition of a 3-manifold $M$ consists of a surface $\Sigma$, with boundary, together with a diffeomorphism $\phi: \Sigma \rightarrow \Sigma$, with $\phi$ the identity near $\partial \Sigma$, such that

$$
M=(\Sigma \times[0,1] / \sim) \cup_{f} \coprod_{i} S^{1} \times D^{2},
$$

where $(x, 1) \sim(\phi(x), 0)$. Note that

$$
\partial(\Sigma \times[0,1] / \sim)=\coprod_{i} T_{i}^{2},
$$

with each torus $T_{i}^{2}$ having a product structure $S^{1} \times[0,1] / \sim$. Let

$$
\lambda_{i}=\{p t\} \times[0,1] / \sim, \quad \lambda_{i} \in T_{i}^{2} .
$$


The gluing diffeomorphism used to construct $M$ is defined by

$$
f: \partial\left(\coprod_{i} S^{1} \times D^{2}\right) \rightarrow \partial\left(\coprod_{i} T_{i}^{2}\right), \quad\{p t\} \times \partial D^{2} \rightarrow \lambda_{i} .
$$

The map $\phi$ is called the monodromy of the open book. See [Etnyre 2006] for more details.

Theorem 2.2 [Alexander 1920]. Every closed oriented 3-manifold has an open book decomposition.

A contact structure $\xi$ on $M$ is said to be supported by an open book decomposition $(\Sigma, \phi)$ of $M$ if $\xi$ can be isotoped through contact structures so that there exists a 1 -form $\alpha$ for $\xi$ such that

- $d \alpha$ is a positive area form on each page, and

- $\alpha(v)>0$ for all $v \in T L$ that induce the orientation on $L$.

Theorem 2.3 [Thurston and Winkelnkemper 1975]. Every open book decomposition $(\Sigma, \phi)$ supports a contact structure $\xi_{\phi}$.

We sketch this well-known proof, as we need the details in later arguments.

Proof. Let

$$
M=(\Sigma \times[0,1] / \sim) \cup_{f} \coprod_{i} S^{1} \times D^{2}
$$

be given as before. We first construct a contact structure on $\Sigma \times[0,1] / \sim$, and then we extend it in a neighborhood of the binding.

In the neighborhood $N=S^{1} \times D^{2}$ of each component of the binding, consider coordinates $(\psi, x, \theta)$, such that $(\psi, x)$ are coordinates on the page (with $\psi$ the coordinate along the binding) and $d \theta$ and $\pi^{*} d \theta$ agree, where $\pi^{*} d \theta$ is the pullback through $\pi: M \backslash L \rightarrow S^{1}$ of the coordinate on $S^{1}$. Let $\lambda$ be a 1 -form on the page that is an element of the set (it is easy to check that this set is not empty)

$$
\begin{array}{r}
S=\{1 \text {-forms } \lambda \text { such that } d \lambda \text { is a volume form on } \Sigma \text { and } \\
\qquad \lambda=(1+x) d \psi \text { near } \partial \Sigma=L\} .
\end{array}
$$

On $\Sigma \times[0,1]$ take $\tilde{\lambda}=(1-\theta) \lambda+\theta\left(\phi^{*} \lambda\right)$ and consider the 1 -form $\alpha_{K}=\tilde{\lambda}+K d \theta$. For sufficiently large $K, \alpha_{K}$ is a contact form and it descends to a contact form on $\Sigma \times[0,1] / \sim$. To extend this form on the solid tori neighborhood of the binding, we pull back $\alpha$ through the gluing map $f$ and get

$$
\alpha_{f}=K d \theta-(x+\epsilon) d \psi \text {. }
$$



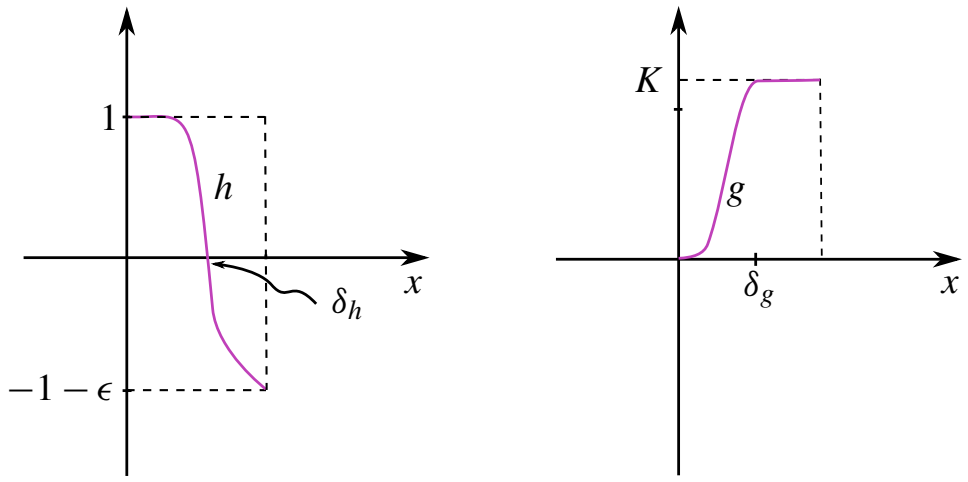

Figure 1. The functions $h$ and $g$.

We are looking to extend this form on the entire $S^{1} \times D^{2}$ to a contact form of the form

$$
h(x) d \psi+g(x) d \theta .
$$

This is possible if there exist functions $h, g:[0,1] \rightarrow \mathbb{R}^{3}$ such that

(i) $h(x) g^{\prime}(x)-h^{\prime}(x) g(x)>0$ (given by the contact condition);

(ii) $h(x)=1$ near $x=0, h(x)=-(x+\epsilon)$ near $x=1$; and

(iii) $g(x)=r^{2}$ near $x=0, g(x)=K$ near $x=1$.

The two functions $h$ and $g$ described in Figure 1 work for our purpose. The conditions (ii) and (iii) are obviously satisfied, and if $\delta_{h}$ and $\delta_{g}$ are such that $h(x)<$ 0 on $\left[\delta_{h}, 1\right]$ and $g(x)=K$ on $\left[\delta_{g}, 1\right]$, then (i) is satisfied as long as $\delta_{g}<\delta_{h}$.

The following correspondence, which is one of the central results in contact geometry, relates open book decompositions and contact structures.

Theorem 2.4 [Giroux 2002]. Let $M$ be a closed, oriented 3-manifold. Then there is a one-to-one correspondence

$$
\left\{\begin{array}{l}
\text { contact structures } \xi \text { on } M^{3} \\
\text { up to contact isotopy }
\end{array}\right\} \stackrel{1-1}{\longleftrightarrow}\left\{\begin{array}{l}
\text { open book decompositions }(\Sigma, \phi) \\
\text { of } M^{3} \text { up to positive stabilization }
\end{array}\right\} .
$$

See [Etnyre 2006] for details.

\section{Braiding knots in contact 3-manifolds}

In this section we generalize the following theorem:

Theorem 3.1 [Bennequin 1983]. Any transverse link $K$ in $\left(\mathbb{R}^{3}, \xi_{\text {std }}\right)$ is transversely isotopic to a link braided about the z-axis. 
Let $(L, \pi)$ be an open book decomposition for $M$. A link $K \subset M$ is said to be braided about $L$ if $K$ is disjoint from $L$ and there exists a parametrization of $K$, $f: \bigsqcup S^{1} \rightarrow M$, such that if $\theta$ is the coordinate on each $S^{1}$ then $(d / d \theta)(\pi \circ f)>0$, for any $\theta$. We call those arcs where this condition is not satisfied bad arcs of $K$.

Theorem 3.2. Suppose $(L, \pi)$ is an open book decomposition for the 3-manifold $M$ and $\xi$ is supported by $(L, \pi)$. Let $K$ be a transverse link in $M$. Then $K$ can be transversely isotoped to a braid.

Proof. The idea of the proof is to find a family of diffeomorphisms of $M$ keeping each page of the open book setwise fixed and sending the parts of the link where the link is not braided into a neighborhood of the binding. A neighborhood of the binding is contactomorphic to a neighborhood of the $z$-axis in $\left(\mathbb{R}^{3}, \xi_{\text {std }}\right) / z \sim z+1$ and there the link can be braided, according to Theorem 3.1. What we really use is that Theorem 3.1 works not only for links, but also for arcs with good ends.

In the neighborhood $N=S^{1} \times D^{2}$ of each component of the binding, consider coordinates $(\psi, x, \theta)$ and let $\lambda \in S$ as described in Theorem 2.3. On $\Sigma \times[0,1]$ take $\tilde{\lambda}=(1-\theta) \lambda+\theta\left(\phi^{*} \lambda\right)$ and consider the family of 1 -forms given by $\alpha_{t}=$ $\tilde{\lambda}-(K / t) d \theta$, where $t \in[-1,0)$ and $K$ is a large constant.

This family of 1-forms descends to a family of 1-forms on $\Sigma \times[0,1] / \sim$ (since $\left.\phi^{*}\left(\left.\alpha_{t}\right|_{\Sigma \times\{0\}}\right)=\left.\alpha_{t}\right|_{\Sigma \times\{1\}}\right)$. Both $\xi_{-1}=\operatorname{ker}\left(\alpha_{-1}\right)$ and $\xi$ are contact structures supported by $(L, \pi)$, and by Giroux's correspondence they are isotopic. Therefore, without loss of generality, we may assume $\xi=\operatorname{ker}\left(\alpha_{-1}\right)$.

For large enough $K$, the family of 1 -forms $\left\{\alpha_{t}\right\}_{t}$ is a family of contact 1-forms:

$$
\alpha_{t} \wedge d \alpha_{t}=\left(\tilde{\lambda}-K \frac{1}{t} d \theta\right) \wedge(d \tilde{\lambda})=\tilde{\lambda} \wedge d \tilde{\lambda}-K \frac{1}{t} d \theta \wedge d \tilde{\lambda}>0 .
$$

Note that $d \tilde{\lambda}$ is an area form on the page, while $d \theta$ vanishes on the page and is positive on the positive normal to the page. This implies that the term that is subtracted is always negative $(t \in[-1,0))$, and therefore $\alpha_{t}$ is a contact form for sufficiently large $K$. We want to extend this family to the whole $M$, so we need to patch in the solid tori neighborhood of the binding. In the neighborhood of the binding we have $\alpha_{t}=(1+x) d \psi-K(1 / t) d \theta$. We pull back this family through the map $f$ used to glue the solid tori in the definition of the open book. With our chosen coordinates we have

$$
f(\psi, x, \theta)=(x-1+\epsilon,-\psi, \theta)
$$

and the pullback family

$$
\alpha_{f, t}=-(x+\epsilon) d \psi-K \frac{1}{t} d \theta .
$$


We are looking to extend these forms on the entire $S^{1} \times D^{2}$ to a family of the form

$$
h_{t}(x) d \psi+g_{t}(x) d \theta .
$$

This is possible if there exist functions $h_{t}, g_{t}:[0,1] \rightarrow \mathbb{R}$ for $t \in[-1,0)$ such that

(i) $h_{t}(x) g_{t}^{\prime}(x)-h_{t}^{\prime}(x) g_{t}(x)>0, t \in[-1,0)$ (given by the contact condition);

(ii) $h_{t}(x)=1$ near $x=0, h_{t}(x)=-(x+\epsilon)$ near $x=1$; and

(iii) $g_{t}(x)=x^{2}$ near $x=0, g_{t}(x)=-K / t$ near $x=1$.
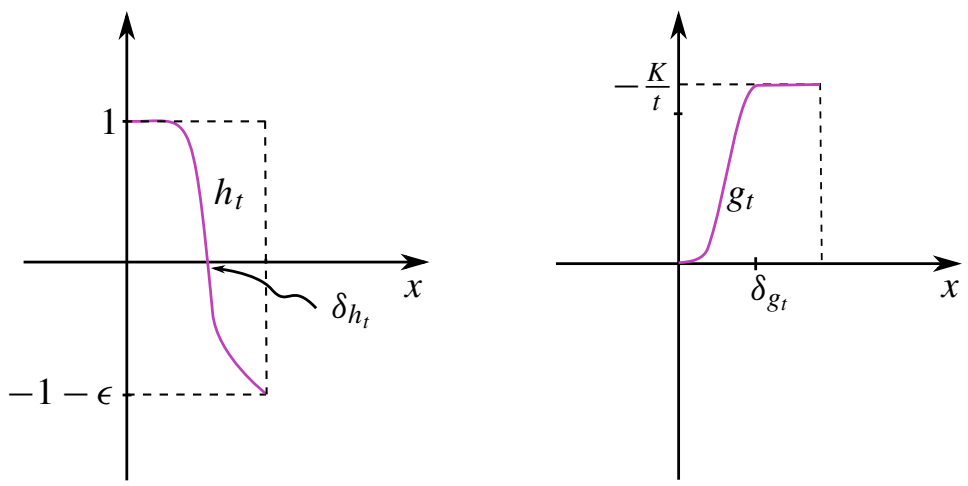

Figure 2. The functions $h_{t}$ and $g_{t}$.

The two families $\left\{h_{t}\right\}_{t \in[-1,0)}$ and $\left\{g_{t}\right\}_{t \in[-1,0)}$ described in Figure 2 work for our purpose. The conditions (ii) and (iii) are satisfied for our choice of $h_{t}$ and $g_{t}$, and if $\delta_{h_{t}}$ and $\delta_{g_{t}}$ are such that $h_{t}(x)<0$ for $x \in\left[\delta_{h_{t}}, 1\right]$ and $g_{t}(x)=-K / t$ for $x \in\left[\delta_{g_{t}}, 1\right]$, then (i) is satisfied as long as $\delta_{g_{t}}<\delta_{h_{t}}$.

Denote the extended family of forms also by $\alpha_{t}$, and by $\xi_{t}$ the family of contact structures given by $\xi_{t}=\operatorname{ker}\left(\alpha_{t}\right)$, with $t \in[-1,0)$. By Gray's theorem, there exists a family of diffeomorphisms $f_{t}: M \rightarrow M$ such that $\left(f_{t}\right)_{*} \xi_{-1}=\xi_{t}$.

As announced, we would like to construct a family of diffeomorphisms $\left\{f_{t}\right\}_{t}$ that fix the pages setwise. Following the proof of Theorem 2.1, we construct $\left\{f_{t}\right\}_{t}$ as the flow of a vector field $X_{t} \in \xi_{t}$, for which we have the equality (2-5).

We already know that such an $X_{t}$ exists, but would need it to be tangent to the page. First notice that

$$
\frac{d \alpha_{t}}{d t}=\frac{1}{t^{2}} K d \theta
$$

and choose some vector $v \in T \Sigma \cap \xi_{t}$. Applying both sides of (2-5) to $v$, we get

$$
d \alpha_{t}\left(X_{t}, v\right)=\frac{d \alpha_{t}}{d t}\left(v_{\alpha_{t}}\right) \alpha_{t}(v)-\frac{d \alpha_{t}}{d t}(v)
$$


As $v \in \xi_{t}=\operatorname{ker}\left(\alpha_{t}\right)$ and $v$ has no $\theta$-component, the last equality is equivalent to

$$
d \alpha_{t}\left(X_{t}, v\right)=0 .
$$

As $d \alpha_{t}$ is an area form on $\xi_{t}$, this implies that $X_{t}$ and $v$ are linearly dependent and therefore

$$
X_{t} \in T \Sigma \cap \xi_{t}
$$

( $X_{t}=0$ at singular points, where $T \Sigma$ and $\xi_{t}$ coincide).

We are now looking at the singularities of $X_{t}, t \in[-1,0)$. Because for $t_{1} \neq t_{2}$, $\alpha_{t_{1}}$ and $\alpha_{t_{2}}$ differ by a multiple of $d \theta$, the flowlines given by $X_{t_{1}}$ and $X_{t_{2}}$ on a page $\Sigma_{\theta}$ coincide. Therefore, it suffices to look at the singularities of $X_{t_{0}}$ for some $t_{0} \in[-1,0)$. On $\Sigma_{\theta}$ there are no negative elliptic singularities away from the binding since the contact planes and the planes tangent to the page almost coincide, as oriented plane fields (a negative elliptic singularity $e$ would require $\xi_{e}$ and $T_{e} \Sigma$ to coincide but have different orientations). Thus, for each $\theta$, all points on $\Sigma_{\theta}$, except for singularities of $X_{t_{0}}$ and stable submanifolds of hyperbolic points, flow in finite time into an arbitrarily small neighborhood of the binding. Define $S_{\theta}$ as the set of points on $\Sigma_{\theta}$ that are either singularities of $X_{t_{0}}$ or on stable submanifolds of hyperbolic points. Let $\mathscr{Y}=\cup S_{\theta}$ as $\theta$ varies from 0 to $2 \pi$.

First, note that we can arrange the monodromy map $\phi$ to fix the singularities on the cutting page, by thinking of $\phi$ as a composition of Dehn twists away from these points. For isolated values of $\theta, X_{t_{0}}$ might exhibit connections between hyperbolic singularities. With that said, $\mathscr{Y}$ has a $C W$ structure with

- as its 1-skeleton, the union of $\{x\} \times[0,1]$ for singular points $x$, and connections between hyperbolic singularities; and

- as its 2-skeleton, the union of stable submanifolds of hyperbolic singularities.

We want to arrange $K$ in such a way that the bad $\operatorname{arcs}$ of $K$ (where $K$ is not braided) are disjoint from $\mathscr{Y}$. Figure 3 depicts a bad $\operatorname{arc}$ of $K$. At the point $p$, which lies on the bad arc, $K$ intersects the contact plane $\xi_{p}$ positively, and it intersects the page negatively (the positive normal vectors to the contact plane and page are shown by arrows). We introduce wrinkles along $K$ as in Figure 3. See below for the explicit definition.
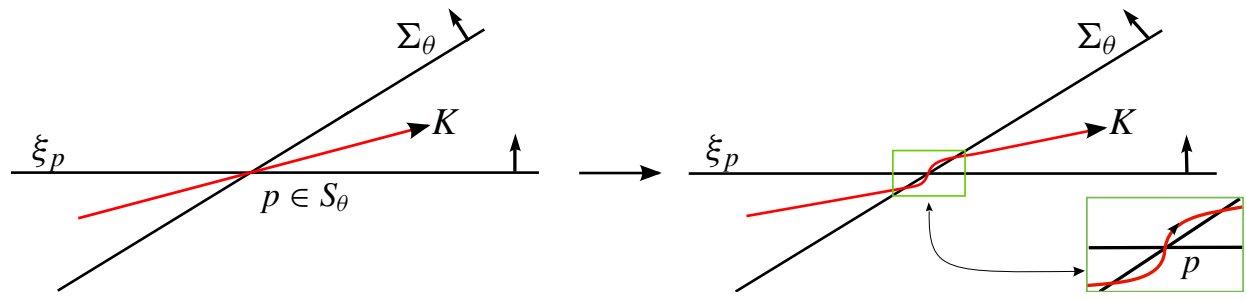

Figure 3. Wrinkling $K$ in order to avoid intersections with $\mathscr{Y}$. 
The wrinkles may increase the number of arcs where the link is not braided, but this is fine, because these new arcs avoid $\mathscr{Y}$.

By general position, we may assume that $K \cap(1$-skeleton of $\mathscr{Y})$ is empty and that $K \pitchfork(2$-skeleton of $\mathscr{Y})$ is a finite number of points. A small neighborhood of $p \in K \pitchfork$ (2-skeleton of $\mathscr{Y})$ in $\Sigma_{\theta}$ is foliated by intervals $(-\epsilon, \epsilon)$, in the same way as a small disk in the $x y$-plane centered at $(0,1,0)$ in $\left(\mathbb{R}^{3}, \xi_{\text {std }}\right)$. It follows that $p$ has a neighborhood in $M$ that is contactomorphic to a neighborhood of $q=(0,1,0)$ in $\left(\mathbb{R}^{3}, \xi_{\text {std }}\right)$. Consider the standard $(x, y, z)$ coordinate system in such a neighborhood of $q$. The contact plane at $q$ is given by the equation $z=x$. Because at $p$ the contact plane and the plane tangent to the page almost coincide, we consider the plane $z=(1+\epsilon) x$ at $q$ to correspond to the plane tangent to the page at $p$. We identify the bad $\operatorname{arc}$ of $K$ with the segment given by

$$
y=1, \quad z=(1+\epsilon / 2) x,
$$

for $x \in[-\delta, \delta]$. We call this segment $W$. See Figure 4. With this setting, the wrinkle takes $W$ to $\tilde{W}$ with the following properties:

(i) $\tilde{W}$ is given by $z=(1+3 \epsilon / 2) x, y=1$ for $x \in(-\delta / 3, \delta / 3)$;

(ii) $\tilde{W}$ is given by $z=(1+\epsilon / 2) x, y=1$ for $x \in[-\delta,-2 \delta / 3) \cup(2 \delta / 3, \delta]$; and (iii) $d z / d x>0$ along $\tilde{W}$ for $x \in(-\delta, \delta)$.
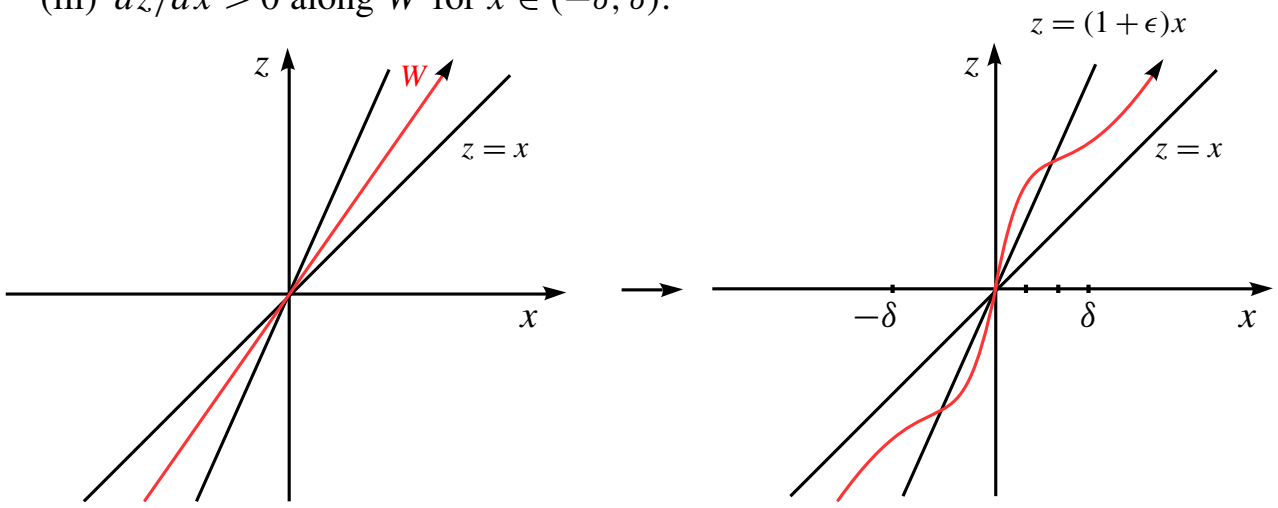

$z=\left(1+\frac{\epsilon}{2}\right) x$

Figure 4. Wrinkling $W$.

Condition (iii) ensures that the link remains transverse throughout the wrinkling. The arc $\tilde{W}$ still intersects the set $\mathscr{Y}$ at the point $q \in \tilde{W}$, but after wrinkling this intersection no longer sits on a bad arc.

We perform this wrinkling at each point where a bad arc of $K$ intersects $\mathscr{Y}$, and obtain a link $\tilde{K}$ that does not intersect $\mathscr{S}$ along any of its bad arcs. We now apply the family of diffeomorphisms $\left\{f_{t}\right\}_{t \in[-1,0)}$. Then $\tilde{K}_{\epsilon}:=f_{\epsilon}(\tilde{K})$ has all its bad arcs in a neighborhood of the binding. By Theorem 3.1, there is a transverse isotopy taking $\tilde{K}_{\epsilon}$ to a braid $B$. This braid $B$ is transversely isotopic to $K$. 


\section{Markov's theorem in an open book decomposition}

The goal of this section is to generalize the following theorem:

Theorem 4.1 [Orevkov and Shevchishin 2003]. In ( $\left.\mathbb{R}^{3}, \xi_{\text {std }}\right)$, two braids represent transversely isotopic links if and only if one can pass from one braid to the other by braid isotopies, positive Markov moves and their inverses.

A Markov move near a component of the binding $L$ is given by the introduction of a new loop around $L$, as in Figure 5. These moves are done near the binding, where the standard coordinates apply. While a positive Markov move (and its inverse) keeps the link transverse, a negative Markov move (and its inverse) fails to do so (see [Bennequin 1983] for details).
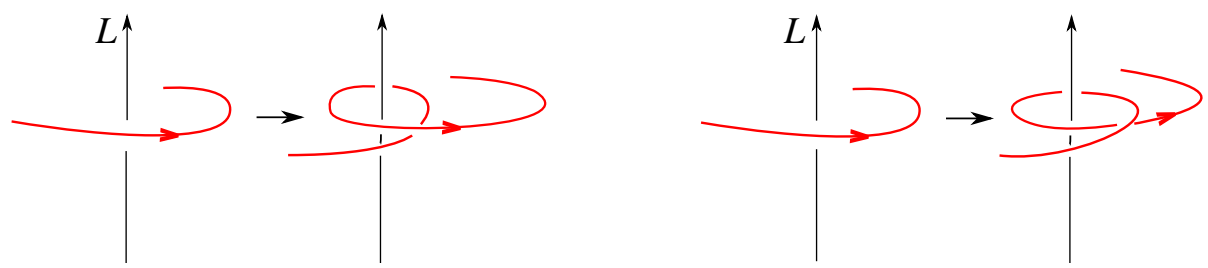

Figure 5. Positive (left) and negative (right) Markov moves.

Theorem 4.2. Let $M$ be a 3-dimensional, closed, oriented manifold and $(L, \pi)$ be an open book decomposition for $M$ together with its supported contact structure $\xi$. Let $K_{0}$ and $K_{1}$ be transverse closed braid representatives of the same topological link. Then $K_{0}$ and $K_{1}$ are transversely isotopic if and only if they differ by braid isotopies and positive Markov moves and their inverses.

The topological version has been previously proven in [Skora 1992] and [Sundheim 1993]. This case immediately follows from the proof of the transverse case, as one does not need to worry about transversality throughout the isotopy. If transversality is not required, both positive and negative Markov moves are allowed.

Proof. The reverse implication is straightforward. An isotopy through braids is done away from the binding. Because the contact planes almost coincide with the planes tangent to the pages, this isotopy stays transverse with respect to the contact structure.

The direct implication takes more work. Let $K_{0}$ and $K_{1}$ be transverse braid representatives of the same topological link $K$, and let $\left\{K_{t}\right\}_{t \in[0,1]}$ be a transverse isotopy from $K_{0}$ to $K_{1}$. We parametrize the isotopy by $\mathscr{K}: \bigsqcup S^{1} \times[0,1] \rightarrow M$, such that $\mathscr{K}_{t}$ defined by $s \rightarrow \mathscr{K}(s, t)$ is a parametrization of $K_{t}$, where $s$ is the positively oriented coordinate on each $S^{1}$. Denote by $\mathfrak{A} \subset M$ the immersed annulus $\mathscr{K}\left(\coprod S^{1} \times[0,1]\right)$. 
Let $\theta$ be the positive coordinate normal to the page. A bad zone of $\mathscr{K}$ is a connected component of $\mathfrak{A}$ where $(\partial / \partial s)(\pi \circ \mathscr{K}) \leq 0$. We denote by $\mathscr{B}$ the union of all bad zones of $\mathscr{K}$.

We wish to take all the bad zones of $\mathscr{K}$ in a neighborhood of the binding. This way, the proof is reduced to the standard case proved in [Orevkov and Shevchishin 2003]. For this, we use the family of diffeomorphisms $\left\{f_{t}\right\}_{t \in[-1,0)}$ constructed in the proof of Theorem 3.2. Keeping the same notations, we want to arrange the isotopy $\mathscr{K}$ in such a way that $\mathscr{B} \cap \mathscr{S}=\varnothing$.

By general position, $\mathscr{B} \cap \mathscr{Y}$ consists of arcs $l$, along which $\partial(\pi \circ \mathscr{K}) / \partial s \leq 0$, and points at which $\partial(\pi \circ \mathscr{K}) / \partial s \leq 0$. The arcs $l$ are intersections of the 2-cells of $\mathscr{Y}$ with $\mathscr{B}$, while the points are intersections of the 1-cells of $\mathscr{G}$ with $\mathscr{B}$. We modify the isotopy $\mathscr{K}$ in such a way as to replace the $\operatorname{arc} l \subset \mathscr{B} \cap \mathscr{S}$ by another $\operatorname{arc} l^{\prime} \subset \mathscr{S}$, along which $\partial(\pi \circ \mathscr{K}) / \partial s>0$, and to eliminate the isolated points of $\mathscr{B} \cap \mathscr{Y}$. We describe the process below.

Let $l \subset \mathscr{B} \cap \mathscr{Y}$, and identify a small region of $\mathscr{B}$ containing $l$ with the rectangle $R=\left[\frac{1}{2}, \frac{3}{2}\right] \times[0,1]$ (denote by $t$ the coordinate on the first factor) such that $l$ is identified with $\left[\frac{1}{2}, \frac{3}{2}\right] \times\left\{\frac{1}{2}\right\}$, and let $R_{t}:=\{t\} \times[0,1] \subset R, t \in\left[\frac{1}{2}, \frac{3}{2}\right]$. We make the following identifications:

- For $t \in\left[\frac{1}{2}, \frac{3}{2}\right]$, identify $R_{t}$ with the segment $W_{t} \subset \mathbb{R}^{3}$ given by $y=t$ and $z=(t+\epsilon / 2) x$.

- For $t \in\left[\frac{1}{2}, \frac{3}{2}\right]$, identify the contact plane at $P\left(t, \frac{1}{2}\right) \in l$ with the contact plane at $(0, t, 0)$, that is, the plane given by $z=t x$.

- For $t \in\left[\frac{1}{2}, \frac{3}{2}\right]$, identify the page of the open book at $\left(t, \frac{1}{2}\right) \in l$ with the plane at $(0, t, 0)$ given by $z=(t+\epsilon) x$.

With these settings, the wrinkle takes $W_{t}$ to $\tilde{W}_{t}, t \in\left[\frac{1}{2}, \frac{3}{2}\right]$, with the following properties:

- $\tilde{W}_{t}$ is given by $z=(t+3 \epsilon / 2) x, y=t$ for $x \in(-\delta / 3, \delta / 3)$.

- $\tilde{W}$ is given by $z=(t+\epsilon / 2) x, y=t$ for $x \in[-\delta,-2 \delta / 3) \cup(2 \delta / 3, \delta]$.

- $d z / d x>0$ along $\tilde{W}_{t}$ for $x \in(-\delta, \delta)$.

See Figure 6.

We perform this wrinkling along every arc in the intersection $\mathscr{B} \cap \mathscr{Y}$. If two such $\operatorname{arcs} l_{1}$ and $l_{2}$ intersect each other, there exist two disjoint regions on $\mathfrak{A}\left(l_{1} \cup l_{2}\right)$, one containing $l_{1}$ and the other containing $l_{2}$, that we can identify with rectangles $R_{1}$ and $R_{2}$ as above. We wrinkle along $l_{1}$ and $l_{2}$ separately.

The wrinkling translates to the original setup as in Figure 7. The bad zone (colored green in Figure 7) no longer intersects $\mathscr{Y}$.

For an isolated point $x \in \mathscr{B} \cap \mathscr{Y}$, we pick a small bad arc $l$ containing $x$ on the isotopy annulus and we do the wrinkling along $l$ as above. 

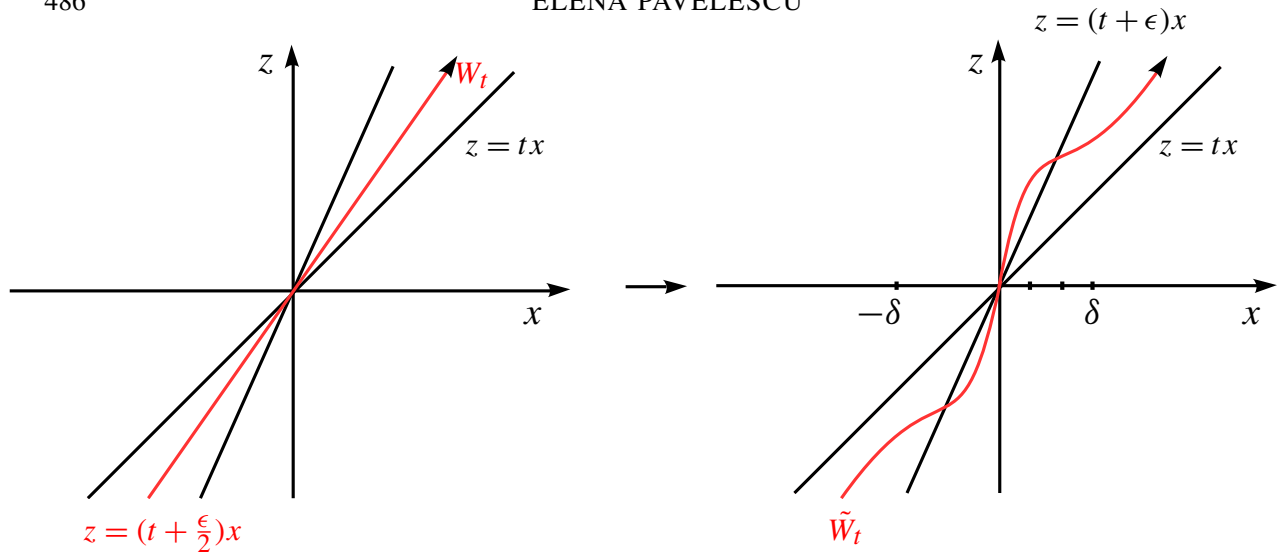

Figure 6. Wrinkling $W_{t}$.
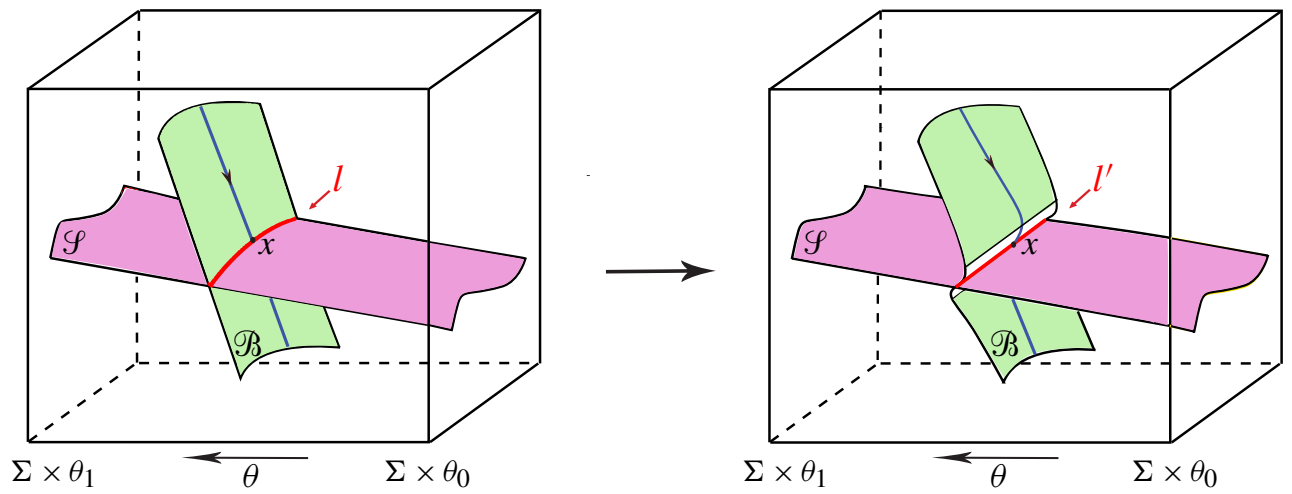

Figure 7. Isotopy modification along an $l$ arc. The new intersection $\operatorname{arc} l^{\prime}$ sits outside of $\mathscr{B}$ (green region).

We obtain an isotopy annulus that does not intersect $\mathscr{Y}$ along any bad arcs.

\section{Acknowledgements}

The author thanks John Etnyre for all his support and guidance. She would also like to thank Evgeny Volkov for taking an interest in these results and pointing out the sign mistake in Theorem 3.2. She thanks Keiko Kawamuro for all the helpful conversations.

\section{References}

[Alexander 1920] J. W. Alexander, "Note on Riemann spaces", Bull. Amer. Math. Soc. 26:8 (1920), 370-372. MR 1560318 JFM 47.0529.02

[Alexander 1923] J. W. Alexander II, “A lemma on systems of knotted curves", Proc. Nat. Acad. Sci. USA 9 (1923), 93-95. JFM 49.0408.03 
[Bennequin 1983] D. Bennequin, "Entrelacements et équations de Pfaff", pp. 87-161 in Third Schnepfenried geometry conference (Schnepfenried, France, 1982), vol. 1, edited by D. Bernard and G. Reeb, Astérisque 107, Soc. Math. France, Paris, 1983. MR 86e:58070 Zbl 0573.58022

[Etnyre 2006] J. B. Etnyre, "Lectures on open book decompositions and contact structures", pp. 103-141 in Floer homology, gauge theory, and low-dimensional topology, edited by D. A. Ellwood et al., Clay Math. Proc. 5, Amer. Math. Soc., Providence, RI, 2006. MR 2007g:57042 Zbl 1108.53050

[Geiges 2008] H. Geiges, An introduction to contact topology, Cambridge Studies in Advanced Mathematics 109, Cambridge University Press, 2008. MR 2008m:57064 Zbl 1153.53002

[Giroux 2002] E. Giroux, "Géométrie de contact: de la dimension trois vers les dimensions supérieures", pp. 405-414 in Proceedings of the International Congress of Mathematicians (Beijing, 2002), vol. 2, edited by T. Li, Higher Ed. Press, Beijing, 2002. MR 2004c:53144 Zbl 1015.53049

[Kawamuro and Pavelescu 2011] K. Kawamuro and E. Pavelescu, "The self-linking number in annulus and pants open book decompositions", Algebr. Geom. Topol. 11:1 (2011), 553-585. MR 2783238 Zbl 05851997

[Markov 1936] A. Markov, "Über die freie Äquivalenz der geschlossenen Zöpfe”, Recueil Math. Moscou, n. Ser. 1 (1936), 73-78. Zbl 0014.04202

[Mitsumatsu 2006] Y. Mitsumatsu, "Convergence of contact structures to foliations", pp. 353-371 in Foliations 2005, edited by P. Walczak et al., World Sci., Hackensack, NJ, 2006. With an appendix by Mitsumatsu and Atsuhide Mori. MR 2007k:53144 Zbl 1222.53090

[Orevkov and Shevchishin 2003] S. Y. Orevkov and V. V. Shevchishin, "Markov theorem for transversal links”, J. Knot Theory Ramifications 12:7 (2003), 905-913. MR 2004j:57011 Zbl 1046. 57005

[Skora 1992] R. K. Skora, "Closed braids in 3-manifolds", Math. Z. 211:2 (1992), 173-187. MR 93m:57009 Zbl 0758.57007

[Sundheim 1993] P. A. Sundheim, "The Alexander and Markov theorems via diagrams for links in 3-manifolds", Trans. Amer. Math. Soc. 337:2 (1993), 591-607. MR 93h:57016 Zbl 0795.57007

[Thurston and Winkelnkemper 1975] W. P. Thurston and H. E. Winkelnkemper, "On the existence of contact forms", Proc. Amer. Math. Soc. 52 (1975), 345-347. MR 51 \#11561 Zbl 0312.53028

[Wrinkle 2002] N. C. Wrinkle, The Markov theorem for transverse knots, thesis, Columbia University, Ann Arbor, MI, 2002, available at http://tinyurl.com/8xh77sd. MR 2703285

Received January 12, 2011.

\author{
ELENA PAVELESCU \\ DEPARTMENT OF MATHEMATICS \\ OCCIDENTAL COLLEGE \\ 1600 CAMPUS ROAD \\ LOS ANGELES 90041 \\ UNITED STATES \\ pavelescu@oxy.edu
}




\title{
PACIFIC JOURNAL OF MATHEMATICS
}

\author{
http://pacificmath.org \\ Founded in 1951 by \\ E. F. Beckenbach (1906-1982) and F. Wolf (1904-1989)
}

\section{EDITORS}

V. S. Varadarajan (Managing Editor)

Department of Mathematics

University of California

Los Angeles, CA 90095-1555

pacific@math.ucla.edu

Vyjayanthi Chari

Department of Mathematics

University of California

Riverside, CA 92521-0135

chari@math.ucr.edu

\section{Robert Finn}

Department of Mathematics Stanford University

Stanford, CA 94305-2125

finn@math.stanford.edu

Kefeng Liu

Department of Mathematics

University of California

Los Angeles, CA 90095-1555

liu@math.ucla.edu
Darren Long

Department of Mathematics

University of California

Santa Barbara, CA 93106-3080

long@math.ucsb.edu

Jiang-Hua Lu

Department of Mathematics

The University of Hong Kong

Pokfulam Rd., Hong Kong jhlu@maths.hku.hk

Alexander Merkurjev

Department of Mathematics

University of California

Los Angeles, CA 90095-1555

merkurev@math.ucla.edu
Sorin Popa

Department of Mathematics University of California

Los Angeles, CA 90095-1555 popa@math.ucla.edu

Jie Qing

Department of Mathematics

University of California

Santa Cruz, CA 95064

qing@cats.ucsc.edu

Jonathan Rogawski

Department of Mathematics

University of California

Los Angeles, CA 90095-1555

jonr@math.ucla.edu

\section{PRODUCTION}

pacific@math.berkeley.edu

\section{SUPPORTING INSTITUTIONS}

ACADEMIA SINICA, TAIPEI

CALIFORNIA INST. OF TECHNOLOGY INST. DE MATEMÁTICA PURA E APLICADA KEIO UNIVERSITY

MATH. SCIENCES RESEARCH INSTITUTE NEW MEXICO STATE UNIV.

OREGON STATE UNIV.

\author{
STANFORD UNIVERSITY \\ UNIV. OF BRITISH COLUMBIA \\ UNIV. OF CALIFORNIA, BERKELEY \\ UNIV. OF CALIFORNIA, DAVIS \\ UNIV. OF CALIFORNIA, LOS ANGELES \\ UNIV. OF CALIFORNIA, RIVERSIDE \\ UNIV. OF CALIFORNIA, SAN DIEGO \\ UNIV. OF CALIF., SANTA BARBARA
}

\author{
UNIV. OF CALIF., SANTA CRUZ \\ UNIV. OF MONTANA \\ UNIV. OF OREGON \\ UNIV. OF SOUTHERN CALIFORNIA \\ UNIV. OF UTAH \\ UNIV. OF WASHINGTON \\ WASHINGTON STATE UNIVERSITY
}

These supporting institutions contribute to the cost of publication of this Journal, but they are not owners or publishers and have no responsibility for its contents or policies.

See inside back cover or pacificmath.org for submission instructions.

The subscription price for 2011 is US \$420/year for the electronic version, and \$485/year for print and electronic.

Subscriptions, requests for back issues from the last three years and changes of subscribers address should be sent to Pacific Journal of Mathematics, P.O. Box 4163, Berkeley, CA 94704-0163, U.S.A. Prior back issues are obtainable from Periodicals Service Company, 11 Main Street, Germantown, NY 12526-5635. The Pacific Journal of Mathematics is indexed by Mathematical Reviews, Zentralblatt MATH, PASCAL CNRS Index, Referativnyi Zhurnal, Current Mathematical Publications and the Science Citation Index.

The Pacific Journal of Mathematics (ISSN 0030-8730) at the University of California, c/o Department of Mathematics, 969 Evans Hall, Berkeley, CA 94720-3840, is published monthly except July and August. Periodical rate postage paid at Berkeley, CA 94704, and additional mailing offices. POSTMASTER: send address changes to Pacific Journal of Mathematics, P.O. Box 4163, Berkeley, CA 94704-0163.

PJM peer review and production are managed by EditFLOW ${ }^{\mathrm{TM}}$ from Mathematical Sciences Publishers.

PUBLISHED BY PACIFIC JOURNAL OF MATHEMATICS

at the University of California, Berkeley 94720-3840

A NON-PROFIT CORPORATION

Typeset in LATEX

Copyright $(2011$ by Pacific Journal of Mathematics 


\section{PACIFIC JOURNAL OF MATHEMATICS}

Volume $253 \quad$ No. $2 \quad$ October 2011

Fusion rules on a parametrized series of graphs

MARTA AsAeda and UfFE HAAGERUP

Group gradings on restricted Cartan-type Lie algebras

YURI BAHTURIN and MIKHAIL KOCHETOV

B2-convexity implies strong and weak lower semicontinuity of partitions of $\mathbb{R}^{n}$

\section{DAVID G. CARABALLO}

Testing the functional equation of a high-degree Euler product

DAVID W. FARMER, NATHAN C. RYAN and RALF SCHMIDT

Asymptotic structure of a Leray solution to the Navier-Stokes flow around a 367 rotating body

Reinhard Farwig, Giovanni P. Galdi and Mads Kyed

Type II almost-homogeneous manifolds of cohomogeneity one

DANIEL GUAN

Cell decompositions of Teichmüller spaces of surfaces with boundary

REN GUO and FENG LUO

A system of third-order differential operators conformally invariant under $\mathfrak{s l}(3, \mathbb{C})$ and $\mathfrak{s o}(8, \mathbb{C})$

Toshinis A KUBO

Axial symmetry and regularity of solutions to an integral equation in a half-space

GUOZHEN LU and JIUYi ZHU

Braiding knots in contact 3-manifolds

\section{ELENA PAVELESCU}

Gradient estimates for positive solutions of the heat equation under geometric flow

JUN SUN 